\title{
Predictors for Progression of Tricuspid Insufficiency Following Left-Sided Valvular Surgery: A Retrospective Cohort Study
}

\author{
Ahmet Aydın, MD, Metin Demircin, MD, Prof, Rıza Doğan, MD, Prof, Mustafa Yılmaz, MD, Prof, \\ İlhan Paşaoğlu, MD, Prof
}

Department of Cardiovascular Surgery, Hacettepe University Medical School, Ankara, Turkey

\section{ABSTRACT}

Background: The operative indications for severe tricuspid insufficiency in patients undergoing left-sided valvular surgery are well defined; however, for mild and moderate insufficiency, the findings are still controversial. In this study, we aimed to document the prognostic parameters for progression of tricuspid insufficiency in patients undergoing left-sided valvular surgery.

Methods: 135 patients undergoing mitral valve \pm coronary bypass surgery were retrospectively examined. Patients with simultaneous tricuspid valve surgery were excluded. Demographic, clinical, and laboratory findings were recorded and the relationship with progression of tricuspid insufficiency was studied.

Results: Valvular pathology was rheumatic in origin in $72.6 \%$ of patients. Tricuspid insufficiency was progressed in $69(51.1 \%)$ of patients, while it did not change in $66(48.9 \%)$ patients. Echocardiographic parameters recorded during follow-up revealed that left ventricular end-diastolic diameter decreased, while there was no significant change in right ventricular end-diastolic diameter. Tricuspid insufficiency was directly related with right ventricular end-diastolic diameter $(P<.05)$, while no relationship was documented with left ventricular end-diastolic diameter. There was a statistically significant correlation between postoperative progression of tricuspid insufficiency and left atrial diameter; however, no relationship was documented with age, sex, valvular disease etiology, comorbidities, or preoperative atrial fibrillation. Risk factor analysis revealed that left atrial diameter was the only prognostic factor.

Conclusion: The indications for mild and moderate tricuspid insufficiency are still not clear for patients undergoing left-sided valvular surgery. In this study, we documented that left atrial dilatation was a prognostic factor for progression of postoperative tricuspid insufficiency. In this group of patients, presence of left atrial dilatation may be an indication for tricuspid valve intervention.

Received fanuary 20, 2019; received in revised form March 12, 2019; accepted March 29, 2019.

Correspondence: Abmet Aydin, MD, Hacettepe University Medical School, Cardiovascular Surgery Department, Sibhiye, Ankara, Turkey; +90-312-30517-73 (e-mail:abmetcamsu@yahoo.com).

\section{INTRODUCTION}

Primary tricuspid insufficiency (TI) is a rare condition. However, secondary or functional TI usually results from mitral and/or aortic valve pathologies. Subsequent pulmonary hypertension in left-sided valve diseases leads to right ventricular dilatation causing tricuspid valve annular dilatation and functional TI [Chan 2009]. Therefore, TI should not be considered as an isolated heart valve pathology, but rather one component of structural right ventricular pathology, as well as a marker for advanced stage of cardiac disease [Beckhoff 2018]. Concomitant tricuspid surgery in patients undergoing left-sided valve surgery is the accepted approach in cases of severe TI. It is a "I C" indication in 2017 ESC/ EACTS guidelines for management of valvular heart disease. However, it is still "II A" indication in patients with mild or moderate insufficiency due to controversial results reported. A tricuspid annular diameter $>40 \mathrm{~mm}$ or $>21 \mathrm{~mm} / \mathrm{m}^{2}$ is the defined parameter [Baumgartner 2017]. Progression of insufficiency is not predictable and there is no consensus on timing for intervention. Age, sex, right ventricular dysfunction, atrial fibrillation, tricuspid annulus diameter, and pulmonary arterial pressure are some of the documented factors influencing the progression soon after left-sided cardiac surgery [Gürsoy 2014]. In this retrospective study, we aimed to determine the risk factors and clinical parameters affecting progression of functional TI following left-sided valve surgery.

\section{MATERIALS AND METHODS}

The study was in compliance with the principles outlined in the Declaration of Helsinki. The data of 344 patients who underwent left-sided valve surgery \pm coronary artery bypass grafting (CABG) between January 2005 and December 2010 were retrospectively collected. Patients with concomitant tricuspid valve surgery $(n=40)$, in-hospital mortality $(n=8)$, lost to follow-up and/or missing follow-up data $(\mathrm{n}=161)$ were excluded. The reason for excluding tricuspid surgery cases is obvious, as the study aimed to follow the natural history of TI. Tricuspid surgery was performed only for severe insufficiency cases. Patients with in-hospital mortality were excluded for the same reason. Patients with CABG surgery were not excluded because they were not isolated CABG cases, rather ones undergoing left-sided valvular surgery. The data of the remaining 135 patients who had regular control 


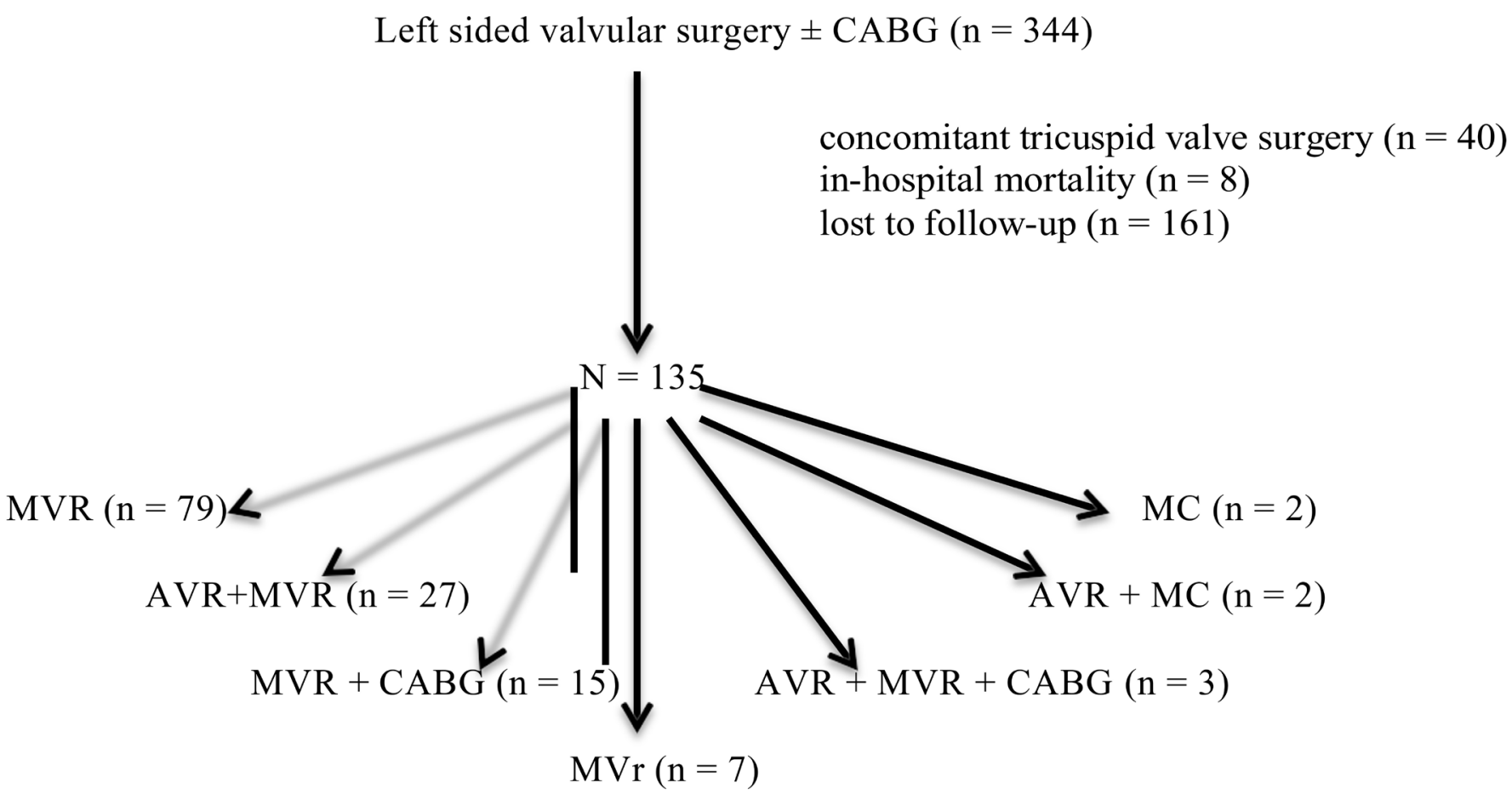

MVR: Mitral Valve Replacement

AVR: Aortic Valve Replacement

\section{CABG: Coronary Artery Bypass Grafting}

Flow chart outlining the study.

visits and follow-up records were analyzed finally. The flow chart for the study is illustrated in the Figure. The demographic, clinical, and laboratory data of all patients were recorded. The patients were evaluated during three different visits: preoperative period, early postoperative period (1 to 6 months), and late postoperative period ( 6 months to 5 years). Routine physical examination together with electrocardiography and echocardiographic examination were performed at all visits.

\section{Statistical Analysis}

Data were analyzed using the Statistical Package for the Social Sciences version 15.0 (SPSS, Chicago, IL, USA). Categorical variables were presented with frequency tables, whereas numerical variables were presented with descriptive statistics (mean, standard deviation, median, minimum, and maximum). Significance level after comparison of independent groups in terms of categorical variables was analyzed using Pearson chi-square test if cross-table statistics provided chi-square condition, or using Monte Carlo simulation if Pearson chi-square condition was not provided. For independent numerical variables, Mann-Whitney $U$ test was used for the comparison of not normally distributed paired groups. For dependent numerical variables, Wilcoxon test was used for the comparison of not normally distributed paired groups,

\author{
MVr: Mitral Valve Repair \\ MC: Mitral Comissurotomy
}

whereas Friedman test was used for multi-group comparisons. Spearman rho coefficient was calculated for the measurement of dependency between not normally distributed groups. Backward stepwise method and logistic regression analysis were used to assess the risk factors. A $P$ value $<.05$ was considered statistically significant.

\section{RESULTS}

The mean age of the patients was $53.4 \pm 13.2$ years and 58 (43\%) were male. The valvular pathology was rheumatic in 98 $(72.6 \%)$ patients whereas degenerative in $20(14.8 \%)$ patients. Only $8(5.9 \%)$ patients had ischemic mitral valve pathology. Among 135 patients, 79 (58.5\%) underwent isolated mitral valve replacement and $27(20 \%)$ underwent both mitral and aortic valve replacement. Baseline demographic, clinical, and surgical characteristics of the patients are represented in detail in Table 1.

Clinical and echocardiographic findings of the patients in preoperative, early postoperative, and late postoperative periods are shown in Table 2. The percentage of patients with NYHA class 3 and 4 was $23.0 \%$ in the preoperative period, $1.5 \%$ in the early postoperative period, and $2.9 \%$ in the late postoperative period $(P<.001)$. The percentage of patients 
Table 1. Demographic Characteristics and Operative Procedures

\begin{tabular}{lc}
\hline & Mean \pm SD \\
\hline Age, $y$ & $53.4 \pm 13.2$ \\
Body surface area, m² & $1.77 \pm 0.19$ \\
& $\mathrm{n}(\%)$ \\
Sex & \\
$<<2$ OPTION SPACES>>Male & $58(43.0)$ \\
<<2 OPTION SPACES>>Female & $77(57.0)$ \\
Chronic obstructive pulmonary disease & $23(17.0)$ \\
Coronary artery disease & $17(12.6)$ \\
Diabetes mellitus & $5(3.7)$ \\
Hypertension & $4(3.0)$ \\
Chronic renal insufficiency & $3(2.2)$ \\
Other & $5(3.7)$ \\
Etiology & \\
Rheumatic valve disease & \\
Degenerative valve disease & $98(72.6)$ \\
Ischemic valve disease & $20(14.8)$ \\
Infective endocarditis & $8(5.9)$ \\
Congenital valve disease & $7(5.2)$ \\
Cardiomyopathy & $1(0.7)$ \\
Operative Procedure & $1(0.7)$ \\
MVR & $27(20.0)$ \\
MVR+AVR & $15(11.1)$ \\
MVR+CABG & $7(5.2)$ \\
MVR+AVR+CABG & $3(2.2)$ \\
\hline & $2(1.5)$ \\
OMC & $2(1.5)$ \\
\hline
\end{tabular}

MVR indicates mitral valve replacement; AVR, aortic valve replacement; CABG, coronary artery bypass; MVP, mitral valvuloplasty; OMC, open mitral commissurotomy.

with severe mitral insufficiency was $50.4 \%$ in the preoperative and zero in early postoperative and late postoperative periods $(P<.001)$. The percentage of those having severe TI was $15.6 \%$ in the preoperative period, whereas it was $14.1 \%$ in the early and $34.8 \%$ in the late postoperative periods $(P<.001)$. The incidence of atrial fibrillation showed no difference between the three time intervals. Mean NYHA class and severity of mitral insufficiency showed a significant decrease in early and late postoperative periods compared to the preoperative period $(P<.001)$. The mean TI degree was similar in the preoperative and early postoperative periods, but it was significantly increased in the late postoperative period $(P<$ .001). Left ventricle end-diastolic diameter (LVEDD), left
Table 2. Preoperative and Postoperative Clinical and Echocardiographic Findings

\begin{tabular}{|c|c|c|c|c|}
\hline & $\begin{array}{c}\mathrm{PrO} \\
\text { mean } \pm \mathrm{SD} \\
(\text { median })\end{array}$ & $\begin{array}{c}\text { PoE } \\
\text { mean } \pm S D \\
(\text { median })\end{array}$ & $\begin{array}{c}\text { PoL } \\
\text { mean } \pm S D \\
\text { (Median) }\end{array}$ & $P$ \\
\hline NYHA Class & $\begin{array}{c}2.01 \pm 0.77 \\
(2) \dagger, \ddagger\end{array}$ & $\begin{array}{c}1.24 \pm 0.47 \\
(1)^{*}, \ddagger\end{array}$ & $\begin{array}{c}1.33 \pm 0.56 \\
(1)^{*}, \dagger\end{array}$ & $<.001$ \\
\hline MI & $\begin{array}{c}2.41 \pm 1.10 \\
(3) \dagger, \ddagger\end{array}$ & $\begin{array}{c}0.22 \pm 0.51 \\
(0)^{*}\end{array}$ & $\begin{array}{c}0.27 \pm 0.57 \\
(0)^{*}\end{array}$ & $<.001$ \\
\hline TI & $1.6 \pm 0.85(2) \ddagger$ & $1.6 \pm 0.87(1) \ddagger$ & $\begin{array}{c}2.19 \pm 0.92 \\
(2)^{*}, \dagger\end{array}$ & $<.001$ \\
\hline MPG, mmHg & $14.80 \pm 9.35 \dagger$, & $9.52 \pm 3.52 *$ & $9.64 \pm 3.46 *$ & $<.001$ \\
\hline MVA, $\mathrm{cm}^{2}$ & $1.83 \pm 1.03 \dagger, \ddagger$ & $2.60 \pm 0.33 *$ & $2.59 \pm 0.34 *$ & $<.001$ \\
\hline LVEDD, cm & $5.35 \pm 0.82 \dagger, \ddagger$ & $4.99 \pm 0.74 *$ & $4.92 \pm 0.75 *$ & $<.001$ \\
\hline RVEDD, cm & $2.53 \pm 0.46$ & $2.51 \pm 0.40 \ddagger$ & $2.6 \pm 0.44 \dagger$ & .003 \\
\hline LAD, cm & $5.01 \pm 0.86 \dagger, \ddagger$ & $4.45 \pm 0.75^{*}$ & $4.39 \pm 0.77^{*}$ & $<.001$ \\
\hline PAP, $\mathrm{mmHg}$ & $\begin{array}{c}48.87 \pm \\
14.06 \dagger, \ddagger\end{array}$ & $\begin{array}{c}35.04 \pm \\
10.12^{*}, \ddagger\end{array}$ & $\begin{array}{c}38.41 \pm \\
10.75^{*}, \dagger\end{array}$ & $<.001$ \\
\hline \multirow[t]{2}{*}{$E F, \%$} & $60.92 \pm 8.71 \dagger$ & $\begin{array}{l}56.53 \pm \\
9.35^{*}, \ddagger\end{array}$ & $60.16 \pm 9.31 \dagger$ & $<.001$ \\
\hline & $n(\%)$ & $n(\%)$ & n (\%) & $P$ \\
\hline $\mathrm{AF}$ & $63(46.7)$ & $64(47.4)$ & $69(51.1)$ & .127 \\
\hline
\end{tabular}

NYHA indicates New York Heart Association; MI, mitral insufficiency; TI, tricuspid insufficiency; MPG, mitral pressure gradient; MVA, mitral valve area; LVEDD, left ventricle end-diastolic diameter; LAD, left atrium diameter; RVEDD, right ventricle end-diastolic diameter; PAP, pulmonary artery pressure; $E F$, ejection fraction; $A F$, atrial fibrillation.

*Different from the preoperative (PrO) period. $\dagger$ Different from the postoperative early (PoE) period. fDifferent from the postoperative late (PoL) period.

atrium diameter, and pulmonary artery pressure values also showed a significant decrease in the early and late postoperative periods compared to the preoperative period $(P<.001)$. Right ventricle end-diastolic diameter (RVEDD) was significantly lower in the early compared to the late postoperative period; however, early and late postoperative period values were not significantly different from preoperative values.

The relation between pre- and postoperative measurements of echocardiography with regard to TI is presented in Table 3. Positive and moderate correlations were found between late postoperative TI and RVEDD. Moreover, a positive and moderate correlation were found between TI and pulmonary artery pressure in the preoperative, early, and late postoperative periods.

The number of patients with increased TI in the late postoperative compared to preoperative period was $69(51.1 \%)$. Characteristics of the groups with $(\mathrm{n}=69)$ and without $(\mathrm{n}=66)$ increase in TI are presented in Table 4 . There was 
Table 3. Pre- and Postoperative Cardiac Measurements in Relation to Tricuspid Insufficiency Degree

\begin{tabular}{|c|c|c|c|c|c|c|c|}
\hline $\mathrm{PrO}$ & $5.23 \pm 0.75$ & $5.43 \pm 0.9$ & $5.36 \pm 0.83$ & $5.19 \pm 0.63$ & - & .626 & -0.042 \\
\hline PoE & $4.98 \pm 0.92$ & $5.04 \pm 0.76$ & $4.97 \pm 0.69$ & $4.98 \pm 0.82$ & $4.5 \pm 0.54$ & .617 & -0.043 \\
\hline PrO & $2.36 \pm 0.37$ & $2.40 \pm 0.39$ & $2.57 \pm 0.48$ & $2.82 \pm 0.46$ & - & $<.001$ & 0.296 \\
\hline PoE & $2.23 \pm 0.31$ & $2.39 \pm 0.32$ & $2.55 \pm 0.37$ & $2.94 \pm 0.38$ & $2.98 \pm 0.69$ & $<.001$ & 0.453 \\
\hline PoL & $2.00 \pm 0$ & $2.32 \pm 0.31$ & $2.47 \pm 0.32$ & $2.87 \pm 0.37$ & $3.20 \pm 0.45$ & $<.001$ & 0.607 \\
\hline \multicolumn{8}{|l|}{ LAD } \\
\hline \multicolumn{8}{|l|}{ PAP } \\
\hline PrO & $38.55 \pm 12.26$ & $42.42 \pm 12.28$ & $54.30 \pm 11.78$ & $57.62 \pm 13.93$ & - & $<.001$ & 0.525 \\
\hline PoE & $25.62 \pm 3.20$ & $31.31 \pm 6.25$ & $36.28 \pm 8.69$ & $48.00 \pm 12.93$ & $47.50 \pm 15.55$ & $<.001$ & 0.543 \\
\hline PoL & $25.00 \pm 0$ & $30.16 \pm 5.40$ & $35.63 \pm 6.11$ & $45.43 \pm 9.88$ & $53.33 \pm 13.87$ & $<.001$ & 0.681 \\
\hline
\end{tabular}

R indicates correlation coefficient (Spearman rho); TI, tricuspid insufficiency; PrO, preoperative period; PoE, postoperative early period; PoL, postoperative late period; LVEDD, left ventricle end-diastolic diameter; RVEDD, right ventricle end-diastolic diameter; LAD, left atrium diameter; PAP, pulmonary artery pressure.

no significant difference between the groups in terms of age, sex, etiology, comorbidities, and the presence of AF in the preoperative period. Echocardiographic measurements of the patients with increased TI in the late postoperative compared to the preoperative periods are presented in Table 5. There was a significant difference only in the left atrial diameter values between groups $(P=.031)$.

Analysis performed to assess the risk factors for progression of TI revealed that left atrial diameter alone was the most significant risk factor in the model created by age, sex, etiology, comorbidities, preoperative atrial fibrillation, mean pulmonary gradient, LVEDD, RVEDD, left atrial diameter, pulmonary artery pressure, and EF. These factors were evaluated in univariate comparisons $[(P=.016 ; \mathrm{OR}=1.704[95.0 \%$ CI 1.103-2.632]).

As the most common etiology was right ventricular dysfunction, pre- and postoperative mean pulmonary gradient values and the mean TI degrees of these patients are presented in Table 6. Patients with right ventricular dysfunction showed a significant decrease in mean pulmonary gradient in the early and late postoperative periods compared to the preoperative period, whereas a significant increase was observed for TI degree $(P<.001)$. The percentage of patients with severe TI was $19.4 \%$ in the preoperative period, $18.4 \%$ in the early postoperative period, and $41.8 \%$ in the late postoperative period.

Furthermore, no ascites were observed in 35 patients having third-degree TI in the late postoperative period, whereas one (2.9\%) had hepatosplenomegaly. Two (16.7\%) of 12 patients having fourth degree TI in the late postoperative period had ascites, whereas $6(50 \%)$ had hepatosplenomegaly. The difference between TI degrees in the late postoperative period and the rate of ascites and hepatosplenomegaly was significant $(P=.021$ and $P<.001$, respectively).

\section{DISCUSSION}

Concomitant tricuspid valve intervention is a widely accepted approach in patients with severe TI undergoing left-sided valve surgery [Baumgartner 2017]. This strategy was reported to be effective and sustainable to eliminate TI and improve right ventricular functions [Bianchi 2009; Desai 2013]. However, the criteria determining the timing of intervention in mild-to-moderate TI remains unclear. As mentioned in 2017 ESC/EACTS guidelines, the objective criteria to indicate surgery is a tricuspid annular diameter $>40 \mathrm{~mm}$ or $>21 \mathrm{~mm} / \mathrm{m}^{2}$ ). However, surgeons still do not always rely on guideline data, instead making decisions based on their experience and status of the patient when indicating and timing surgery. Risk factors for progression of TI following mitral valve surgery include age, sex, right ventricular dysfunction, pulmonary hypertension, tricuspid annular dilatation, and atrial fibrillation [Bianchi 2009]. It was stated that the primary risk factor for progression is tricuspid annular dilatation, and surgical intervention should depend on annular dimensions 
Table 4. Characteristics of the Groups with and without an Increase in Tricuspid Insufficiency Degree in the Postoperative Late Period Compared to the Preoperative Period

\begin{tabular}{|c|c|c|c|}
\hline & $\begin{array}{l}\text { Increase in TI } \\
\quad(n=69) \\
\text { mean } \pm S D\end{array}$ & $\begin{array}{c}\text { No increase in } \mathrm{TI} \\
(\mathrm{n}=66) \\
\text { mean } \pm S D\end{array}$ & $P$ \\
\hline \multirow[t]{2}{*}{ Age, y } & $53.8 \pm 13.1$ & $52.9 \pm 13.4$ & .698 \\
\hline & $\mathrm{n}(\%)$ & $\mathrm{n}(\%)$ & \\
\hline \multicolumn{4}{|l|}{ Sex } \\
\hline Male & $30(43.5)$ & $28(42.4)$ & .902 \\
\hline Female & $39(56.5)$ & $38(57.6)$ & \\
\hline \multicolumn{4}{|l|}{ Etiology } \\
\hline Rheumatic valvular disease & $54(78.3)$ & $44(66.7)$ & .278 \\
\hline Degenerative valve disease & $7(10.1)$ & $13(19.7)$ & \\
\hline Ischemic valve disease & $4(5.8)$ & $4(6.1)$ & \\
\hline Infective endocarditis & $2(2.9)$ & $5(7.6)$ & \\
\hline Congenital valve disease & $1(1.4)$ & $0(0.0)$ & \\
\hline Cardiomyopathy & $1(1.4)$ & - & \\
\hline COPD & $16(23.2)$ & $7(10.6)$ & .076 \\
\hline Coronary artery disease & $7(10.1)$ & $10(15.2)$ & \\
\hline Diabetes mellitus & $4(5.8)$ & $1(1.5)$ & \\
\hline Hypertension & $4(5.8)$ & - & \\
\hline Chronic renal insufficiency & $1(1.4)$ & $2(3.0)$ & \\
\hline Other & $2(2.9)$ & $3(4.5)$ & \\
\hline Preoperative AF & $32(46.4)$ & $37(53.6)$ & .945 \\
\hline
\end{tabular}

TI indicates tricuspid insufficiency; COPD, chronic obstructive pulmonary disease; $\mathrm{AF}$, atrial fibrillation.

[Baumgartner 2017]. A study comprising 959 patients with mild-to-moderate functional TI undergoing mitral valve surgery concluded that early or late clinical benefits of concomitant tricuspid valve repair were uncertain when patients with and without concomitant repair were compared [Ro 2013]. Further studies are needed to determine risk factors. In the present study, we retrospectively analyzed the risk factors influencing the progression of functional TI at follow-up and aimed to document prognostic factors, of which left atrial diameter was promising.

Echocardiography is widely used for the evaluation of valvular pathologies. It was reported that measurement of the tricuspid annulus diameter with two-dimensional echocardiography may reveal differences, since the leaflets of the valve are not on the same plane anatomically. Today, along with the advanced technology and accumulation of knowledge, it is known that real-time three-dimensional (real time-3D) echocardiography and magnetic resonance imaging (MRI) provide more accurate results about tricuspid annulus measurement. Vena contracta width, proximal isovelocity surface
Table 5. Echocardiographic Measurements of the Groups with and without an Increase in Tricuspid Insufficiency Degree in the Postoperative Late Period Compared to the Preoperative Period

\begin{tabular}{lccc}
\hline & $\begin{array}{c}\text { Increased TI } \\
(\mathrm{n}=69) \\
\text { mean } \pm \text { SD }\end{array}$ & $\begin{array}{c}\text { No increase in TI } \\
(\mathrm{n}=66) \\
\text { mean } \pm \text { SD }\end{array}$ & $P$ \\
\hline MPG, mmHg & $15.58 \pm 9.98$ & $13.99 \pm 8.64$ & .422 \\
LVEDD, cm & $5.31 \pm 0.87$ & $5.40 \pm 0.78$ & .439 \\
RVEDD, cm & $2.51 \pm 0.44$ & $2.55 \pm 0.47$ & .775 \\
LAD, cm & $5.17 \pm 0.89$ & $4.84 \pm 0.80$ & .031 \\
PAP, mmHg & $47.38 \pm 15.12$ & $50.42 \pm 12.79$ & .096 \\
EF, \% & $62.07 \pm 8.36$ & $59.71 \pm 8.97$ & .201 \\
\hline
\end{tabular}

TI indicates tricuspid insufficiency gradient; MPG, mitral pressure gradient; LVEDD, left ventricle end-diastolic diameter; LAD, left atrium diameter; RVEDD, right ventricle end-diastolic diameter; PAP, pulmonary artery pressure; $E F$, ejection fraction.

area, effective regurgitant area, regurgitation volume, and tricuspid annular excursion plane measurements are more reliant [Beckhoff 2018; Vahanian 2018]. However, these methods cannot be used in every patient due to high costs and availability. In this present study as well, we evaluated the patients only with two-dimensional echocardiography and used tricuspid insufficiency grading from 1 to 4 .

Tricuspid insufficiency usually occurs due to right ventricular dysfunction and annular dilatation secondary to other valve pathologies rather than primary abnormality of itself. Annular dilatation of tricuspid valve is a more reliable parameter than the degree of TI [Beckhoff 2018; Baumgartner 2017]. Since the present study was retrospective and tricuspid annular measurements were not routinely performed in our clinic, tricuspid annulus diameter could not be evaluated; only degree of TI was recorded. From our point of view, the indications for performing or not performing tricuspid intervention cannot be clearly made. Different operators at different time intervals also make this harder. But still, we believe that an increase in right ventricular dimensions indirectly indicates the increase in annular diameter. In the present study, the mean LVEDD was significantly decreased in early and late postoperative periods compared to the preoperative period. The mean RVEDD was significantly lower in the early rather than the late postoperative period, whereas no difference was observed between postoperative and preoperative values. Nevertheless, it was determined that TI increased in direct proportion to the increase in RVEDD.

Evaluation of tricuspid annulus diameter and fractional shortening of the annulus are the two defined parameters more significant than the degree of insufficiency [Beckhoff 2018]. In a retrospective analysis of 96 patients with 2+ TI, it was documented that insufficiency significantly improved following mitral valve surgery, but soon progressed in mid 
Table 6. Pre- and Postoperative Mitral Pressure Gradient Values and Tricuspid Insufficiency Degrees of the Patients with Rheumatic Valvular Disease

\begin{tabular}{lcccc}
\hline & PrO & PoE & PoL \\
& mean \pm SD (median) & mean \pm SD (median) & mean \pm SD (median) & $P$ \\
\hline MPG, mmHg & $18.52 \pm 7.71(18) \dagger$, $\neq$ & $9.47 \pm 3.31(9)^{*}$ & $9.67 \pm 3.54(9)^{*}$ & $<.001$ \\
TI degree & $1.68 \pm 0.86(2) \dagger, \ddagger$ & $1.72 \pm 0.88(2)^{*}$ & $2.34 \pm 0.92(2)^{*}$ & $<.001$ \\
\hline
\end{tabular}

TI indicates tricuspid insufficiency; MPG, mitral pressure gradient; PrO, preoperative period; PoE, postoperative early period; PoL, postoperative late period.

*Different from the preoperative (PrO) period.

$\dagger$ Different from the postoperative early (PoE) period.

$\ddagger$ Different from the postoperative late (PoL) period.

to late term follow-up over 7 years. The analysis also defined a tricuspid annular diameter $>21.2$ as a cut-off value for TI progression [Kusajima 2016]. In the present study, a decrease in TI degree was observed in the early postoperative period compared to the preoperative period, similar to those findings. While the ratio of patients with third and fourth degree TI was $23 \%$ in preoperative period, it regressed to $1.5 \%$ in the early postoperative period. No significant difference was found between mean TI degrees in the early postoperative and preoperative periods $(1.6 \pm 0.85$ and $1.6 \pm 0.87$, respectively). However, the mean TI degree $(2.19 \pm 0.92)$ in the late postoperative period was significantly higher than in the early postoperative and preoperative periods. The ratio of patients with third and fourth degree TI was significantly increased (up to $34.8 \%$ ) in the late postoperative period.

Left atrial dilatation and an increase in pressure may lead to atrial fibrillation. It causes right atrial dilatation and thereby tricuspid annular dilatation [Beckhoff 2018; Vahanian 2018]. Thus, atrial fibrillation following mitral valve surgery has been reported as an important risk factor for the development or progression of TI [Takano 2017]. Preoperative atrial fibrillation was an independent predictor for late TI development. The present study found no difference among preoperative, early and late postoperative periods in terms of the presence of AF. There was also no significant difference between the groups with and without an increase in TI in the late postoperative period in terms of preoperative atrial fibrillation.

When prognostic significance of functional TI was explored in a study of 1194 patients, it was documented that left atrial diameter had prognostic importance to reach the primary endpoints of cardiac death, admission due to heart failure, and mitral or tricuspid valve intervention [Abe 2018]. That study design was not similar to ours, as they followed up patients with preserved left ventricular functions and atrial fibrillation; but the result was statistically very significant as increased left atrial diameter caused progression in TI and increased primary endpoints. In patients undergoing aortic valve replacement, left atrial diameter was also reported to be a prognostic factor for progression of functional TI [Yajima 2018]. Conversely, in a study aimed to document a tricuspid annular circumference index, there was no correlation between patients with a left atrial diameter $<60 \mathrm{~mm}$ and
$>60 \mathrm{~mm}$. The study stated that left atrial diameter was not a marker to show TI progression [Popal 2018].

Tricuspid regurgitation after mitral valve replacement in right ventricular dysfunction is a serious clinical problem. If dysfunction is severe, it is unlikely that tricuspid intervention will affect the prognosis [Kammerlander 2014]. In the present study, the etiology was right ventricular dysfunction in $98(72.6 \%)$ patients. The ratio of severe (third and fourth degree) TI among those with right ventricular dysfunction was $19.4 \%$ in the preoperative period, whereas it was $41.8 \%$ in the late postoperative periods. $54(78.3 \%)$ of 69 patients with an increase in TI degree in the late postoperative period were patients with right ventricular dysfunction.

It has been reported that pulmonary artery pressure is decreased after a successful mitral valve replacement or balloon valvuloplasty in patients with severe pulmonary hypertension [Beckhoff 2018; Baumgartner 2017; Vahanian 2018; Kammerlander 2014]. Sudden decrease in pulmonary artery pressure after a successful procedure results from elimination of pathology that accounts for the passive component of pulmonary hypertension [Vahanian 2018]. Persisting pulmonary hypertension in such patients would further decrease, along with the decrease in reactive pulmonary arterial vasoconstriction, in a week and in subsequent months. While such a decrease is more remarkable in young non-chronic patients, it is encountered less in old patients with chronic pulmonary hypertension [Kammerlander 2014]. In the present study, we observed that pulmonary artery pressure was significantly decreased in the early and late postoperative periods compared to the preoperative period. Tricuspid insufficiency regressed in the early postoperative period but soon increased in the late postoperative period despite low pulmonary artery pressure. Nevertheless, the direct proportion between pulmonary artery pressure and TI degrees aroused interest when the intergroup analysis was performed.

In the present study, the analyses performed to determine the risk factors for progression of functional TI following mitral valve surgery revealed left atrial diameter alone as the most significant risk factor in the model created by the following factors: age, sex, etiology, comorbidities, preoperative atrial fibrillation, mean pulmonary gradient, LVEDD, RVEDD, left atrial diameter, pulmonary artery pressure, and EF. 
In a meta-analysis of 2448 patients, those undergoing tricuspid valve annuloplasty were compared with those not in cases of left sided valvular surgery and mild to moderate functional TI. Performing tricuspid intervention concomitantly significantly reduced progression of TI and suggested a more aggressive surgical approach [Kara 2015]. Similarly, another meta-analysis with 2840 patients concluded that tricuspid valve repair strategy concomitant with left-sided valve surgery decreased cardiac related mortality and degree of TI during follow-up [Pagnesi 2017]. We may not be able to make such a conclusion from our study, but we also support this approach based on the data recently published irrespective of sophisticated echocardiographic measurements.

In conclusion, LEVDD was increased, whereas the mean RVEDD showed no significant change in the postoperative period. Tricuspid insufficiency degree increased in direct proportion to RVEDD, but LVEDD was not associated with TI. No significant relationship was found between preoperative atrial fibrillation and TI progression. This can be attributed to its already existing effects on tricuspid valve and atrium. We suggest that the occurrence of atrial fibrillation following mitral valve surgery might enhance TI. Risk factor analysis revealed that left atrial diameter was the only factor influencing the progression of TI at follow-up. To the best of our knowledge, there are limited data that would guide to what extent TI would be improved after repair of the left-sided valve pathologies. There is also no definite satisfactory method that would measure TI and right ventricular functions in acceptable ranges. Large-scale randomized studies with longer follow-up periods are needed on this subject. Preoperative evaluation of patients who would undergo mitral valve surgery should be performed thoroughly, risk factors should be identified, annular diameter, ventricle and atrium diameters should be measured precisely with echocardiography, and patients should be evaluated by real-time 3D echocardiography or MRI when required. As a result, we can say that we believe that patients with mitral valve surgery will benefit from interfering with tricuspid annuloplasty if left atrial dilation has occurred, even if TI is not serious.

\section{Study Limitations}

The study is retrospective in origin, and therefore some data more valuable today was not recorded at that time, such as sophisticated tricuspid regurgitation echocardiographic parameters (vena contracta width, proximal isovelocity surface area, effective regurgitant area, regurgitation volume, and tricuspid annular excursion plane). If available, the data would be more reliable. Moreover, despite tricuspid annular diameter measurements, degree of TI was the preferred parameter of choice, which may be regarded as insufficient or even wrong. The operations were performed by different surgeons at different time intervals; therefore, clear indications for performing or not performing tricuspid intervention could not be clearly made.

\section{REFERENCES}

Abe Y, Akamatsu K, Ito K, et al. 2018. Prevalence and prognostic significance of functional mitral and tricuspid regurgitation despite preserved left ventricular ejection fraction in atrial fibrillation patients. Circ J 82:1451-8.

Baumgartner H, Falk W, Bax J, et al. 2017.2017 ESC/EACTS Guidelines for the management of valvular heart disease. Eur Heart J 38:2739-91.

Beckhoff F, Alushi B, Jung C, et al. 2018. Tricuspid regurgitation-medical management and evolving interventional concepts. Front Cardiovasc Med 5:49.

Bianchi G, Solinas M, Bevilacqua S, Glauber M. 2009. Which patient undergoing mitral valve surgery should also have the tricuspid repair? Interact Cardiovasc Thorac Surg 9:1009-20.

Chan KM, Zakkar M, Amirak E, Punjabi PP. 2009. Tricuspid valve disease: pathophysiology and optimal management. Prog Cardiovasc Dis 51:482-6.

Desai RR, Vargas Abello LM, Klein AL, et al. 2013. Tricuspid regurgitation and right ventricular function after mitral valve surgery with or without concomitant tricuspid valve procedure. J Thorac Cardiovasc Surg 146:1126-32.

Gürsoy M, Bakuy V, Hatemi AC, et al. 2014. Long-term prognosis of mild functional tricuspid regurgitation after mitral valve replacement. Anatol J Cardiol 14:34-9.

Kammerlander AA, Marzluf BA, Graf A, et al. 2014. Right ventricular dysfunction, but not tricuspid regurgitation, is associated with outcome late after left heart valve procedure. J Am Coll Cardiol 64:2633-42.

Kara I, Koksal C, Erkin A, et al. 2015. Outcomes of mild to moderate functional tricuspid regurgitation in patients undergoing mitral valve operations: A meta-analysis of 2,488 patients. Annals Thorac Surg 100:2398-2407.

Kusajima K, Fujita T, Hata H, Shimahara Y, Miura S, Kobayashi J. 2016. Long-term echocardiographic follow-up of untreated 2+ functional tricuspid regurgitation in patients undergoing mitral valve surgery. Interact Cardiovasc Thorac Surg 23:96-103.

Pagnesi M, Montalto C, Mangieri A, et al. 2017. Tricuspid annuloplasty versus a conservative approach in patients with functional tricuspid regurgitation undergoing left-sided heart valve surgery: A study-level meta-analysis. Int J Cardiol 240:138-44.

Popal MS, Fu JT, Hu QM, Luo TG, Zheng S, Meng X. 2018. Intraoperative method based on tricuspid annular circumference in patients with mild or no tricuspid regurgitation during left-sided cardiac valve surgery for the prophylactic tricuspid annuloplasty. J Thorac Dis 10:3670-8.

Ro SK, Kim JB, Jung SH, Choo SJ, Chung CH, Lee JW. 2013. Mildto-moderate functional tricuspid regurgitation in patients undergoing mitral valve surgery. J Thorac Cardiovasc Surg 146:1092-7.

Takano H, Miramatsu M, Kida H, et al. 2017. Severe tricuspid regurgitation after mitral valve surgery: the risk factors and results of the aggressive application of prophylactic tricuspid valve repair. Surg Today 47:445-56.

Vahanian A, Brochet E, Juliard JM. 2018. Guidelines recommendations on the treatment of tricuspid regurgitation. Where are we and where do we go with transcatheter valve intervention. Front Cardiovasc Med 5:37.

Yajima S, Yoshioka D, Toda K, et al. 2018. Definitive determinant of late significant tricuspid regurgitation after aortic valve replacement. Circ J 82:886-94. 